\title{
Sialendoscopy Training: Presentation of a Realistic Model
}

\author{
Gabriela Robaskewicz Pascoto ${ }^{1}$ Aldo Cassol Stamm ${ }^{2}$ Marcos Lyra ${ }^{3}$ \\ ${ }^{1}$ Department of Otolaryngology, Avita Medicina, Florianópolis, \\ Santa Catarina, Brazil \\ 2 Department of Otorhinolaryngology, Complexo Hospitalar Edmundo \\ Vasconcelos, São Paulo, Brazil \\ ${ }^{3}$ Department of Gynecology, Universidade Federal de Pernambuco,

\begin{abstract}
Address for correspondence Gabriela Robaskewicz Pascoto, MD, Vasconcelos, Rua Borges Lagoa 1450, São Paulo 04038-905, Brazil (e-mail: bls_gaby@yahoo.com.br).
\end{abstract} \\ Department of Otorhinolaryngology, Complexo Hospitalar Edmundo
} Recife, Pernambuco, Brazil

Int Arch Otorhinolaryngol 2017;21:17-20.

\begin{abstract}
Keywords

- salivary glands

- learning

- medical education

Introduction Several surgical training simulators have been created for residents and young surgeons to gain experience with surgical procedures. Laboratory training is fundamental for acquiring familiarity with the techniques of surgery and skill in handing instruments.

Objective The aim of this study is to present a novel simulator for training sialendoscopy.

Method This realistic simulator was built with a synthetic thermo-retractile, thermosensible rubber which, when combined with different polymers, produces more than 30 different formulas. These formulas present textures, consistencies, and mechanical resistance are similar to many human tissues.

Results The authors present a training model to practice sialendoscopy. All aspects of the procedure are simulated: month opening, dilatation of papillae, insert of the scope, visualization of stones, extraction of these stones with grasping or baskets, and finally, stone fragmentation with holmium laser.

Conclusion This anatomical model for sialendoscopy training should be considerably useful to abbreviate the learning curve during the qualification of young surgeons while minimizing the consequences of technical errors.
\end{abstract}

\section{Introduction}

To improve the learning curve of residents, several surgical training simulators have been created, especially in neurosurgery and plastic surgery areas. ${ }^{1,2}$ Those surgical simulators have an important role in training, given the potentially dire consequences of technique errors. ${ }^{1}$

Among the several methods performed by otolaryngologists is the sialendoscopy - the endoscopy of salivary glands. ${ }^{3}$ Despite sialendoscopy being a thirty-year-old technique, few surgeons know how to perform and deal with the delicate instruments. Laboratory training is fundamental for acquiring familiarity with the techniques of surgery and skill in handling instruments. ${ }^{1,2}$

received

September 14, 2015

accepted

June 18, 2016

published online

August 16, 2016
Some courses have been organized around the world to teach sialendoscopy techniques. The current most commonly used model in such courses is a pig head, which provides the training in the papilla of salivary ducts very well. However, this model clearly has limitations.

First of all, the pig's salivary glands do not present the pathologies, such as stones and strictures.

Second, the use of animal models can raise notable ethical objections and is even forbidden is some countries. Otherwise, the manipulation of animals is not allowed anywhere. Many sanitary laws may be respected to have such animals for training.

Third, due to the post mortem rigor, is almost impossible to perform sialendoscopy in fresh human cadavers. In few hours the mandibular jaw cannot be easily opened. ${ }^{4,5}$

Copyright $\odot 2017$ by Thieme-Revinter Publicações Ltda, Rio de Janeiro, Brazil 


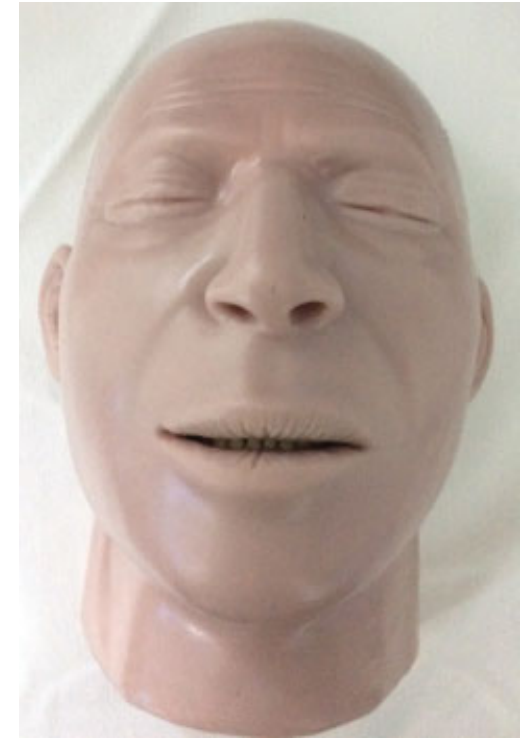

Fig. 1 MAX simulator.

\section{Objective}

With the objective to solve these problems, the goal of this report is to present a realistic simulator for training sialendoscopy.

\section{Method}

\section{Description of the Simulator}

The authors, together with the Brazilian company ProDelphus (www.prodelphus.com), have worked on creating and developing a new synthetic model that could provide a reliable model for sialendoscopy called MAX sialendoscopy.

This realistic simulator was built with a synthetic thermoretractile, thermo-sensible rubber called Neoderma ${ }^{\circledR}$ (ProDelphus, Olinda, Brazil) which, when combined with different polymers, produces more than 30 different formulas. These formulas present textures, consistencies, and mechanical resistance similar to many human tissues.

A special resin, in the shape of the anterior part of skull, constitute the basic structure of MAX sialendoscopy (-Fig. 1).

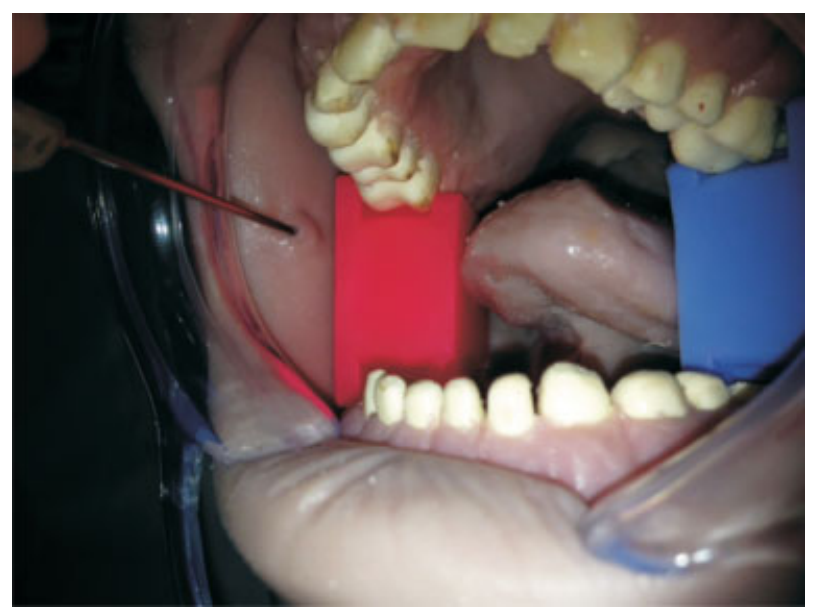

Fig. 3 Dilatation of right parotid papilla.

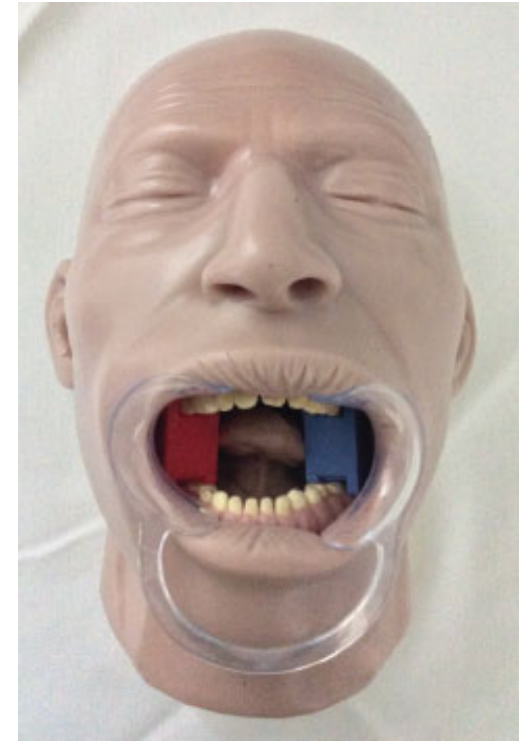

Fig. 2 MAX with mouth openers.

The model contains an articular joint of mandibular bone which allows the month opening (-Fig. 2). Inside the oral cavity, it is possible to notice many realistic structures, such as tongue, teeth, uvula, and tonsils. The papillae of both parotid and submandibular glands are located in realistic sites.

Due to all this anatomical specificity, the surgeon can fully perform sialendoscopy procedure and techniques.

\section{Results}

The authors hereby present a training model to practice the sialendoscopy. It was possible to simulate all aspects of the procedure, from patient mouth opening to extracting stones from the salivary ducts.

The experienced surgeons could observe multiple opportunities for practice, such as dilatation of papillae ( - Figs. 3 and 4), insertion of the scope ( - Fig.5), visualization

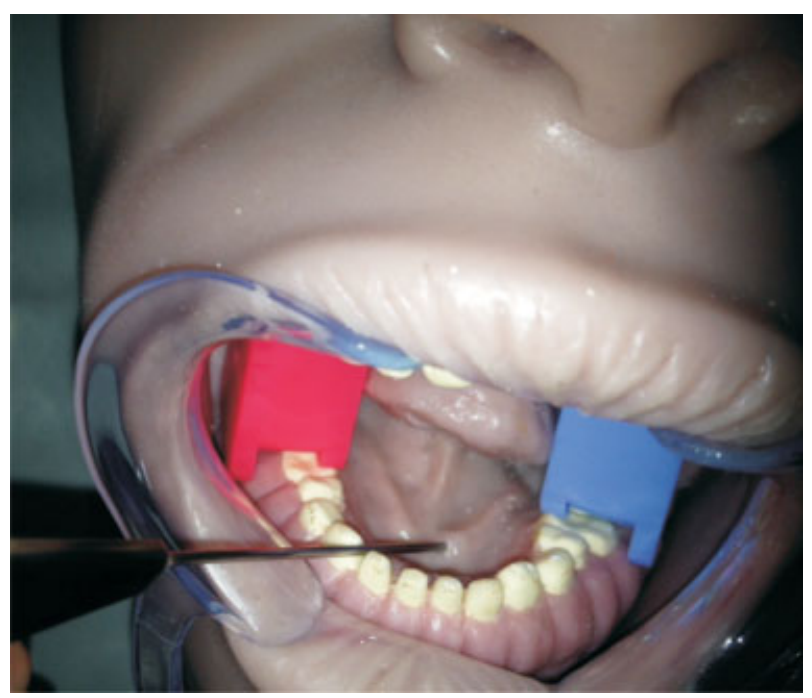

Fig. 4 Dilatation of left submandibular papilla. 


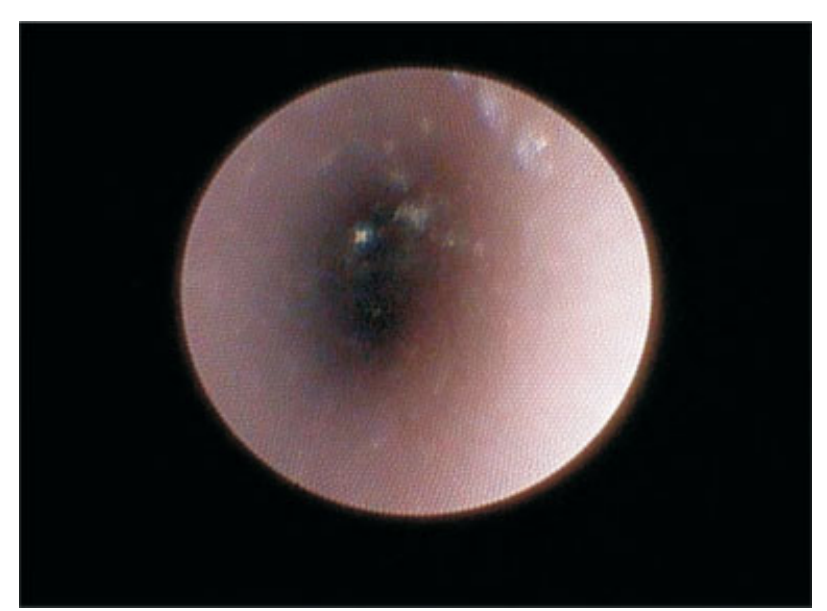

Fig. 5 Main salivary duct vision.

of stones (-Figs. 6 and 7 ), extraction of the stones with grasping or baskets ( - Fig. 8), and finally, stone fragmentation with holmium laser.

\section{Discussion}

The treatment of many salivary ducts' pathologies using the technique of sialendoscopy express a challenge for the surgeon. All anatomical structures involved are delicate and once a rude movement is done, the entire procedure may be impaired. In any case, all instruments are delicate and the training to improve skills in handling them is the key for a successful sialendoscopy. In this context, the training based on surgical simulation is extremely helpful.

Many models have been created as the initial step in training residents and young surgeons to gain experience with surgical procedures. $^{1,2}$

Previous investigators have reported class I data that supports the ability of simulators to improve performance in the operating room (OR). ${ }^{6-8}$

The use of realistic simulators can reduce the errors, maximize the sensitivity in demonstrating skills transfer, and improve the learning curve prior to undertaking the real surgery. ${ }^{1}$

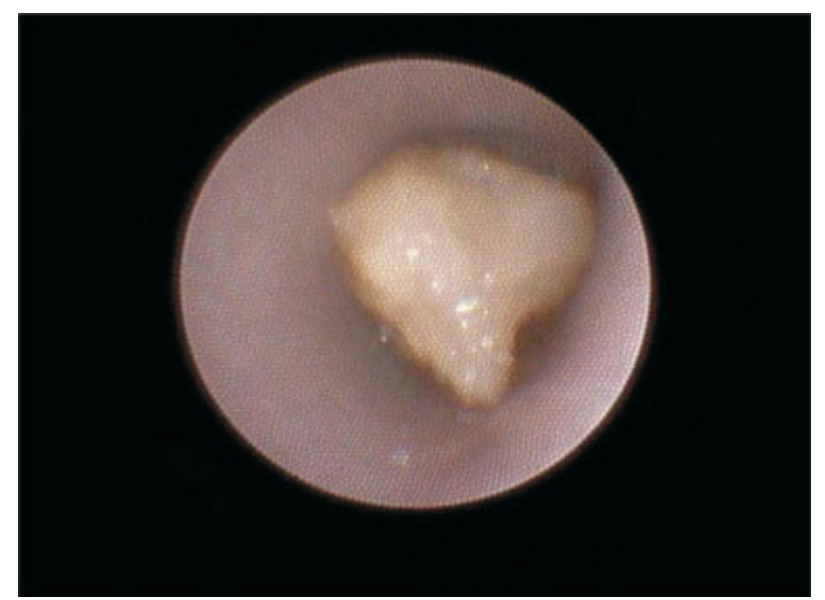

Fig. 7 Stone trapped.

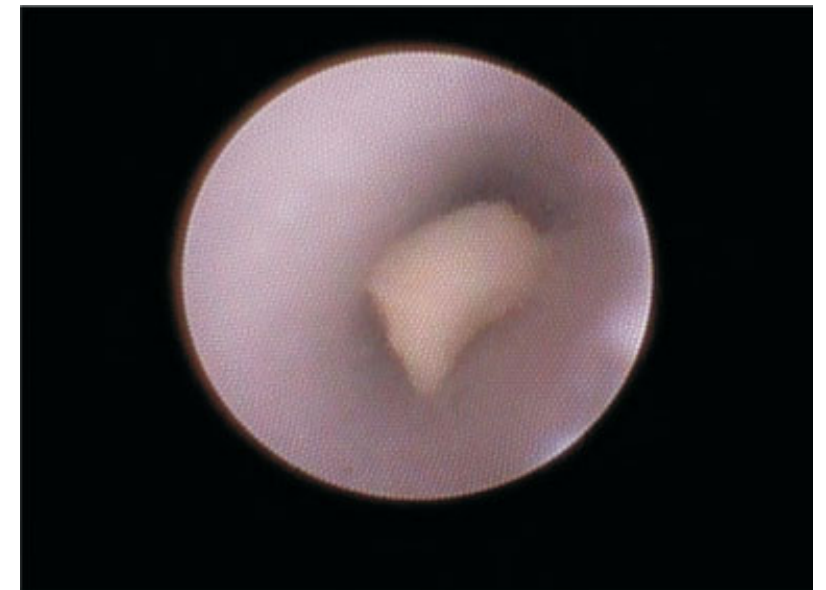

Fig. 6 Floating stone in the salivary duct.

In this respect, our MAX sialendoscopy simulator is not only the first realistic simulator available for sialendoscopy, but also provides an alternative to the use of animal models and human cadaveric specimens.

Moreover, this simulator presents other advantages:

1. The material maintenance is convenient, and there is no need for any kind of special preparation. It is easier to maintain when compared with animal models.

2. The model expresses the appropriate size and the dimensions observed in salivary ducts. Thereby, the identification of anatomical landmarks and, more importantly, the depth perception is feasible.

3. This real simulator includes all necessary planning steps to perform sialendoscopy.

4. The ability to obtain realistic imaging studies provides knowledge of the pathological condition and its diagnosis.

5. There are not ethical restrictions to its use or the need for a specific place to work with the simulator.

6. "Easy logistics": possibility to organize courses and send models worldwide - no need for special care and/or legal authorizations.

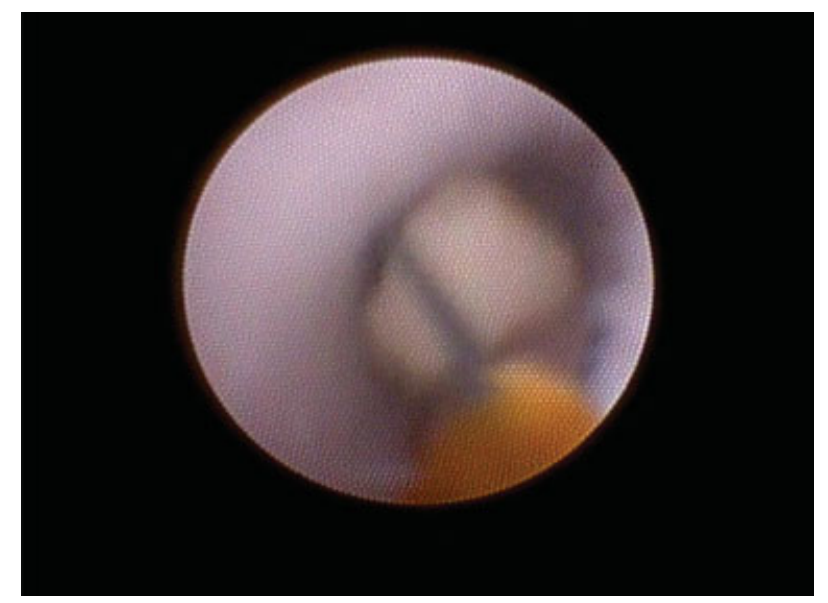

Fig. 8 Using a basket to remove the stone. 
On the other hand, it is important to emphasize some disadvantages of this simulator. Although the handmade and hand-tuned process of developing such a simulator has the most preferable characteristics (to represent the tissue properties adequately and to express the anatomical structures with precise localization), it can be difficult and extremely time consuming to create.

Another factor that must be considered is the estimated cost for each surgical unit. The single pig cranium, when compared with this realistic simulator, is far less expensive.

However, we stress that the benefits of such models, including their reproducibility of emergency situations, may be superior in their ability to augment the training of surgical residents. Prospective studies will be necessary to validate the effectiveness of this training model.

\section{Conclusion}

This report not only represents the first description of the anatomical model for sialendoscopy training but also reinforces its promising prospects. It should be considerably useful to abbreviate the learning curve during the qualification of young surgeons while minimizing the consequences of technical errors.

Future studies will address the scientific validation using well-defined performance measures, possibly followed by integration of this new educational tool into the otorhinolaryngology curriculum. We believe that this teaching method will provide the ability to train out the learning curve for technical skills on a simulator rather than on a patient.

\section{References}

1 Coelho G, Warf B, Lyra M, Zanon N. Anatomical pediatric model for craniosynostosis surgical training. Childs Nerv Syst 2014;30(12): 2009-2014 10.1007/s00381-014-2537-x

2 Zymberg S, Vaz-Guimarães Filho F, Lyra M. Neuroendoscopic training: presentation of a new real simulator. Minim Invasive Neurosurg 2010;53(1):44-46 10.1055/s-0029-1246169

3 Ziegler CM, Steveling H, Seubert M, Mühling J. Endoscopy: a minimally invasive procedure for diagnosis and treatment of diseases of the salivary glands. Six years of practical experience. $\mathrm{Br} \mathrm{J}$ Oral Maxillofac Surg 2004;42(1):1-7 10.1016/s0266-4356(03)00188-8

4 Nakayama Y, Aoki Y, Niitsu H, Saigusa K. Forced oral opening for cadavers with rigor mortis: two approaches for the myotomy on the temporal muscles. Forensic Sci Int 2001;118(1):37-42

5 Shapiro HA. Rigor mortis. BMJ 1950;2(4673):304

6 Lehmann KS, Ritz JP, Maass H, et al. A prospective randomized study to test the transfer of basic psychomotor skills from virtual reality to physical reality in a comparable training setting. Ann Surg 2005;241(3):442-449

7 Palter VN, Grantcharov T, Harvey A, Macrae HM. Ex vivo technical skills training transfers to the operating room and enhances cognitive learning: a randomized controlled trial. Ann Surg 2011;253(5):886-889

8 Seymour NE, Gallagher AG, Roman SA, et al. Virtual reality training improves operating room performance: results of a randomized, double-blinded study. Ann Surg 2002;236(4):458-463, discussion 463-464 